\title{
EL COOPERATIVISMO EN LA EDUCACIÓN
}

\author{
POR \\ Elena INGLADA GALIANA ${ }^{1}$, \\ José Manuel SASTRE CENTENO ${ }^{2}$ y \\ Begoña VILLARROYA LEQUERICAONANDIA ${ }^{3}$
}

\section{RESUMEN}

Este trabajo plantea la hipótesis de que las cooperativas de enseñanza conforman una alternativa sólida para el futuro de los centros de enseñanza españoles. Cierto que ya son una realidad en este momento coyuntural, pero los retos con que se enfrenta el sector de la enseñanza en un futuro próximo y a medio plazo, pueden ser afrontados con mayores posibilidades de ofrecer calidad, eficiencia y, en conjunto, excelencia desde las perspectivas de un planteamiento ideológico, profesional y económico como el que ofrecen las cooperativas de la enseñanza.

Para demostrar la hipótesis de trabajo se ha desarrollado, en una primera parte de este artículo, un sucinto repaso a la historia de las cooperativas de enseñanza desde los primeros pasos en los albores del siglo XX hasta nuestros días especialmente los relacionados con su evolución en España.

En la segunda parte se han analizado la estructura, características y problemáticas de dichas cooperativas de enseñanza y su proyección dentro de un mundo caracterizado por la globalización, las nuevas tecnologías, la información y el conocimiento que inciden, directamente, en la enseñanza.

Todo ello conduce a pronosticar un futuro inmediato en que las cooperativas de enseñanza se impondrán, vistas las premisas de este trabajo, a los centros privados de los que son, ya hoy día, una alternativa que se consolidará en el futuro.

\footnotetext{
${ }^{1}$ Universidad de Valladolid. Dirección de correo electrónico: elenaig@eco.uva.es

${ }^{2}$ Universidad de Valladolid. Dirección de correo electrónico: $\underline{\text { manolo@eco.uva.es }}$

${ }^{3}$ Universidad de Valladolid. Dirección de correo electrónico: begovi@eco.uva.es

REVESCO No 118 - Segundo Cuatrimestre 2015 - ISSN: 1885-8031 - www.ucm.es/info/revesco

http://dx.doi.org/10.5209/rev_REVE.2015.n118.49059

Fecha de recepción: 27/09/2013

Fecha de aceptación: 11/11/2014
} 
Palabras clave: Democracia, socio, alternativa, sociedad, ideología, empatía.

Claves Econlit: P100, I200, I280.

\title{
THE COOPERATIVE MOVEMENT IN EDUCATION
}

\begin{abstract}
This work hypothesizes that teaching cooperatives provide a solid alternative for the future of the Spanish teaching centers. Certainly they are a reality related to the moment, but the challenges facing the education sector in the near and half future, may be faced with a greater chance of providing quality, efficiency and overall, from the perspectives of excellence based in an ideological, economic and professional approach such as that as offered by teaching cooperatives.
\end{abstract}

To demonstrate the working hypothesis, in the first part of this article a concise overview of the history of teaching cooperatives has been developed from first steps at the dawn of the 20th century until our days specially those related to its evolution in Spain.

In the second part we analyze the structure, characteristics and problems in those teaching cooperatives and its projection in a world characterized by globalization, new technologies, information and knowledge all of which impacts directly on teaching

All of this leads to predict in the immediate future that teaching cooperatives will be imposed, and given the assumptions of this paper mainly in the private centers where they are actually an alternative which will be consolidated in the future.

Keywords: Democracy, social, alternative, society, ideology, empathy

\section{INTRODUCCIÓN}

El futuro de las cooperativas de la enseñanza en el siglo XXI deviene tanto de su historia, la ideología cooperativista que comportan, como de las estructuras organizativas que se han desarrollado en los últimos años y que, todos estos factores juntos, comportan una diferenciación clara con los centros de enseñanza privada convencionales.

Esta diferenciación les otorga una ventaja competitiva que les sitúa en mejor posición para afrontar este futuro que se prevé. Esta es la hipótesis sobre la que se ha desarrollado este 
trabajo. Esta ventaja competitiva no ha sido tanto buscada como consecuencia de los factores antes mencionados y su evolución a lo largo del siglo XX.

Para demostrar esta hipótesis de trabajo se han marcado como objetivos desarrollar los principales acontecimientos históricos que han conformado las modernas cooperativas de enseñanza, tanto en el mundo como en España; analizar la organización y estructura de dichas cooperativas con el objeto de resaltar sus valores o defectos; estudiar las características de las mismas, su problemática específica, los tipos de cooperativa de enseñanza y su incidencia en el futuro de dichas cooperativas.

Para alcanzar estos objetivos que fundamentan la hipótesis establecida, se ha desarrollado una metodología de tipo cualitativo cuyo principal investigación ha sido la búsqueda bibliográfica y en hemerotecas, así como en la red a través de portales y bancos de datos relacionados con el tema. También se ha trabajado con archivos y documentos de organizaciones e instituciones públicas y privadas que disponen de información relacionada con las cooperativas.

La importancia de la enseñanza en el la sociedad actual tanto desde las posiciones del Estado como de los padres y profesionales, así como sus proyecciones sobre el futuro, parecen suficiente justificación para desarrollar un estudio sobre este tema.

\section{EVOLUCIÓN HISTÓRICA}

El cooperativismo nace en el siglo XIX en Europa y como consecuencia de la Revolución Industrial ${ }^{4}$. Las dificultades de todo tipo con las que se enfrentaba el obrero sólo podían solucionarse en parte sobre la base de una cooperación entre ellos. El concepto cooperación podemos definirlo siguiendo a Marx como: "La forma de trabajo de muchos obreros coordinados y reunidos con arreglo a un plan en el mismo proceso de producción o en procesos de producción distintos, pero enlazados.” (Marx, 1973, pág. 281).

Las ventajas de todo tipo que para el proletario ofrecía la cooperativa fue la causa de que las nuevas fórmulas se expandieran rápidamente y estructuraran uno de los dos sistemas de apoyo y solidaridad sobre los que se asentó la clase obrera: el sindicato que les defendía en

\footnotetext{
4 “Los hombres de ciencia entienden por revolución industrial el proceso del paso del trabajo manual, en el oficio y la manufactura, a la producción maquinizada (fabril). La principal consecuencia social de este proceso fue la formación de las dos clases fundamentales de la sociedad burguesa (la burguesía industrial y el proletariado fabril) y el surgimiento de la lucha entre ellas" (Mijailov, 1984, pág. 9)
} 
el orden político y la cooperativa que lo hacía en el orden económico. (Rodríguez González, 2009).

Cierto que podemos encontrar antecedentes incluso entre los artesanos del Antiguo Egipto y a través de todas las civilizaciones que han existido hasta llegar a la nuevas, porque el cooperativismo aparece en determinadas coyunturas, pero también es cierto que no todos los modelos que podemos reclamar como antecedentes y tildar de precooperativos son parecidos a los que, a partir del siglo XIX, se desarrollan en Europa y otros países de Occidente.

En la base de este cooperativismo que nace de la Revolución Industrial, se halla el socialismo utópico que es el primer desarrollo de la moderna ideología socialista. La denominación es de Marx, que la opuso al socialismo científico. En la esencia del movimiento está la evolución frente a la revolución, el pacifismo frente a la violencia y el entendimiento con las otras clases sociales, lo que les diferencia claramente del marxismo. Los nombres de las diversas tendencias de este primer socialismo y sus ideas cooperativistas son ahora referencias más icónicas que doctrinales: Robert Owen, Charles Fourier, Saint- Simón, Phillipe Buchez, Michel Derrion o Louis Blanc (Pedro Núñez, 2007).

Como hemos dicho el movimiento cooperativo se extendió, especialmente a partir de los últimos años del siglo XIX y el XX, por todo el mundo, calculándose que hoy día casi 800.000 personas forman parte del sistema cooperativo (Amesti, 2005).

El movimiento cooperativo, en el siglo XX, está enfocado al desarrollo de la economía al servicio de la humanidad, a la satisfacción de las necesidades y a implementar los valores y principios que aparecen en la Declaración de Identidad de la Alianza Cooperativa Internacional ${ }^{5}$.

La misma ACI da una definición de cooperativa que reúne las características esenciales de la misma: "Una cooperativa es una asociación autónoma de personas que se han unido voluntariamente para hacer frente a sus necesidades y aspiraciones económicas,

\footnotetext{
${ }^{5}$ Dicha declaración dice sobre los valores de la Alianza Cooperativa Internacional (ACI): Las cooperativas se basan en los valores de autoayuda, auto responsabilidad, democracia, igualdad, equidad y solidaridad. En la tradición de los fundadores, los socios de las cooperativas sostiene los valores éticos de honestidad, apertura, responsabilidad social y preocupación por los demás.(Journal International Association of Cooperative Law, núm. 25, págs.. 21-26).
} 
sociales y culturales comunes por medio de una empresa de propiedad conjunta y democráticamente controlada."6

\subsection{En el Mundo}

Una cooperativa de enseñanza no es muy diferente de cualquier otro tipo de cooperativa, únicamente que tiene unos objetivos esenciales para la sociedad y para el individuo como son formar a las nuevas generaciones. Por ello podemos afirmar que un centro de enseñanza es una cooperativa cuando se rige por los principios, legislación y normativas del cooperativismo. Dicha organización se estructura alrededor de un grupo de profesores que se une para establecer su propia empresa de enseñanza, con lo que se crean una serie de puestos de trabajo controlados por los cooperativistas, se aplica un ideario consensuado, ofrece una enseñanza de calidad y se asegura el futuro de los profesionales.(Fuentes Viñas, 2002)

Y aunque podemos remontar los antecedentes de las cooperativas de enseñanza a los primeros tiempos del cooperativismo, en la seguridad que siempre encontraríamos ejemplos más o menos fehacientes entre los socialistas utópicos o los socialistas cristianos, que no siempre se diferenciaban claramente, en los siglos XVIII y XIX, no es objetivo de este trabajo la historia del movimiento cooperativista general, por lo que nos ceñiremos a los primeros desarrollos de las cooperativas en la enseñanza que pueden considerarse como tales y son inmediatas precursoras de las actuales.

En 1919, y en buena parte como consecuencia del final de la I Guerra Mundial, surge en Francia la primera Cooperativa escolar de la mano de Bartolomé Profit, un inspector de Enseñanza Elemental, con el objetivo de ayudar en la reconstrucción de las escuelas en su país después de la conflagración y proveerlas del material necesario. El éxito de la idea fue inmediato y en 1923 existían miles de cooperativas de la enseñanza en Francia, país que en 1928 crea la Office Central de la Coopération a 1'Ecole, que coordina el movimiento y le proporciona las normativas legales necesarias. A partir de estos años, el movimiento se expande por todo el mundo afianzándose en el sector educativo de gran número de países.

\footnotetext{
${ }^{6}$ La ley 27/1999 de Cooperativas, define a estas como: "La cooperativa es una sociedad constituida por personas que se asocian, en régimen de libre adhesión y baja voluntaria, para la realización de actividades empresariales, encaminadas a satisfacer sus necesidades y aspiraciones económicas y sociales, con estructura y funcionamiento democrático, conforme a los principios formulados por la Alianza Cooperativa Internacional en los términos resultantes de la presente Ley."
} 
El especial desarrollo de la primera mitad del siglo XX, que terminó en el estallido de la II Guerra Mundial, impidió una acción conjunta e internacional del cooperativismo durante muchos años, hasta que en 1964 aparece la Comisión Internacional de la Cooperación Escolar de la que fue primer presidente Maurice Colombain, vinculado con la Organización Internacional del Trabajo (OIT), que en su ideario consideraba que la cooperativa de enseñanza representaba una herramienta de producción eficaz y económica que permitía obtener un mayor valor educativo por la sociedad a un coste razonable y, por otra parte, el mismo proceso cooperativo aplicado a la enseñanza era altamente educativo (1950). El proceso de expansión del cooperativismo en la etapa final del siglo XX se acelera y en 1971 ya abarca los cinco continentes y 67 países.

Un año después, George Prevot, reforma la Comisión Internacional de la Cooperación Escolar que se denomina a partir de este momento Bureau International de la Coopératión Scolaire (BICS) y más tarde Madelaine Alary, en 1984, la transforma en la Alliance International de la Coopération Scolaire (AICS) que prepara la inserción del cooperativismo escolar en las organizaciones transnacionales. Estos tres nombres y estas tres instituciones fundamentaron el nuevo cooperativismo escolar en el ámbito internacional (Arango Jaramillo, 2005).

\subsection{En España}

"Son cooperativas de enseñanza las que desarrollan actividades docentes, en sus distintos niveles y modalidades. Podrán realizar también, como complementarias, actividades extraescolares y conexas, así como prestar servicios que faciliten las actividades docentes." (Ley 27/1999, de 16 julio, de Cooperativas).

Las primeras experiencias de cooperativismo en la enseñanza aparecen en España en la década de los sesenta del pasado siglo XX en las regiones más industrializadas: País Vasco y Cataluña. Estos primeros intentos son promovidos por padres de alumnos que no están de acuerdo con el tipo de enseñanza que se da en los centros educativos del momento, controlados por la ideología del régimen franquista o por la de la Iglesia Católica que domina el sector en aquellos años.

La primera experiencia que a la larga se consolidó fue la cooperativa Ulgor que aparece en 1956 junto con el Grupo Mondragón Corporación Cooperativa, bajo la dirección 
del sacerdote José María Arizmendiarrieta, que poco después a finales de la misma década apadrina la Liga de Educación y Cultura que terminaría siendo una cooperativa.

Dicha Liga, cuyo territorio de actuación era la comarca del Alto Deba, en Guipúzcoa, estaba integrada por centros de distintos niveles de educación y fue sostenida por diversas instituciones públicas y privadas de la región.

Una de las características de estas primeras cooperativas, que después se reprodujo en otras posteriores pero no en todas, fue la creación del binomio estudio-trabajo de manera que se orientaba la educación hacia la técnica y las necesidades de la industria, en algunos casos como en el de la Cooperativa Mondragón la propia.

El cooperativismo en la enseñanza en Cataluña habrá de esperar hasta 1966 en que se crea la Escuela Vilarnau en San Sadurni d'Anoia y poco después la Escuela Montbau de Barcelona. En los dos casos, tanto en el País Vasco como en Catalunya, dejando de lado las razones de tipo ideológico, existían fuertes razones de tipo económico que impulsaban la creación de cooperativas. La crisis de los años setenta obligó a los productores de amplios sectores a hacerse cargo de empresas en quiebra o crear otras nuevas para defender puestos de trabajo. Lo cual se mezcló con otros ideales. (Ormaetxea, 2003)

En dicha década de los setenta, el cooperativismo español en general y el cooperativismo de la enseñanza en particular, siguiendo las tendencias de la sociedad adquirió perfiles políticos y, en algunas autonomías, nacionalista, aun cuando también ofrecían un nuevo modelo de enseñanza que era una auténtica alternativa a la educación tradicional y religiosa. Incorporarán modelos pedagógicos que se estaban siguiendo en países avanzados en esta especialidad, más progresistas y acordes con los conceptos de libre enseñanza. Todo ello comportó que las cooperativas de enseñanza devienen en la vanguardia de los movimientos sociales y reivindicativos, eso sí, con las tendencias nacionalistas que hemos apuntado.

El impulso para el salto cualitativo y cuantitativo que se dio a partir de esta década, lo provocaron por una parte los padres y por otra los profesores o socios trabajadores que en algunos casos por su cuenta en otros asociados, crearon cooperativas más o menos autónomas. En el conjunto del territorio español se impusieron las cooperativas organizadas por los profesores en un $90 \%$ de los casos, excepto en el País Vasco donde se dio más la cooperativa mixta de padres de alumnos y profesionales.(Fuente Viñas, 2002). 
Para Moreno Hernández (1992) el éxito de las cooperativas en esta etapa de finales de los setenta y toda la década de los ochenta, se debe a la crisis de la enseñanza privada del momento, que fue ocasionada por:

.- Recesión económica que afecta la economía de los padres.

- Mejoría en la calidad de la enseñanza estatal, especialmente en lo que a nuevos y modernos centros se refiere y, también, en la renovación pedagógica.

-- Mejor control por parte de la Administración Central de los centros, que se produce a causa de las subvenciones económicas que aumentan en esta época y exigen más inspecciones.

.- Un aumento en la demanda de plazas escolares debido a ciclos demográficos.

Todo ello provoca que la patronal de la enseñanza privada, ante las pérdidas económicas que se produjeron, cierre centros y venda edificios dedicados a la enseñanza. La Administración Central no puede asumir el aumento de la demanda de plazas y adopta una política de impedir el cierre de los centros de enseñanza promoviendo que sean los propios trabajadores de dichos centros quienes se agrupen en cooperativas para hacerse cargo de los mismos. Dándose la paradoja que el propio régimen franquista, a través del Decreto 488/1973 de 1 de marzo, prioriza las ayudas y beneficios a las cooperativas de enseñanza de padres de alumnos o profesores equiparándolas a las instituciones religiosas y públicas.

En 1987 aparece la Ley 3/1987 de 2 de abril, general de Cooperativas, que más tarde, en 1999 sería sustituida por la 27/1999. En el artículo 145 de la ley de 1987, se definen por primera vez las cooperativas de enseñanza en una ley, artículo que hemos reproducido al principio de este apartado. 
Gráfico 1. Situación cuantitativa de las Cooperativas Españolas de Enseñanza - 2012

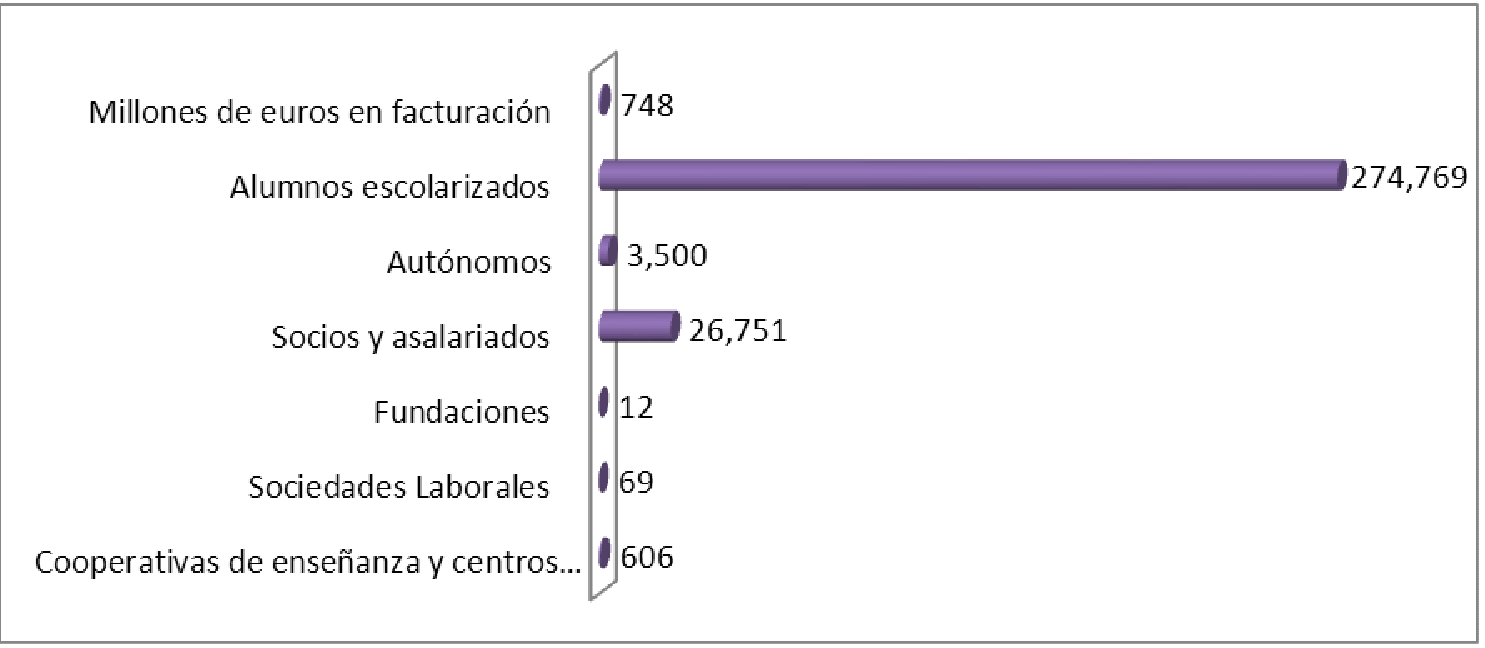

Fuente: Elaboración propia a partir de los datos de la UECOE

Evidentemente muchas de las cooperativas que surgen en estos años no son fundadas por una ideología, por implantar un modelo pedagógico o por adoptar los conceptos y principios del movimiento cooperativista internacional, sino por la necesidad de defender los puestos de trabajo o las plazas de los alumnos. En números globales, de las 640 cooperativas de enseñanza que existen en las décadas de los ochenta y noventa en España, la mayoría, 458 (un $71 \%$ ), corresponden a cooperativas de trabajo asociado creadas por los profesionales, 120 las organizadas por los padres y unas 62 mixtas, aunque estas últimas tienden a aumentar por la fusión de parte de los dos grupos anteriores. El panorama respondía a una necesidad claramente económica y profesional, no tanto a una idea docente, aun cuando el resultado fuera potenciar esta última.

Y con este bagaje se entra, con respecto al tema que analizamos, en el siglo XXI, que desde los primeros años del mismo implicará una fuerte reestructuración del sector. Ello se debe a varios factores que se acumulan en todo el espectro educativo español. En primer lugar aumenta el período de enseñanza obligatorio, lo que significa un aumento de estudiantes en las escuelas y, consecuentemente, un aumento de los profesionales dedicados a la enseñanza y, más concretamente, de los cooperativistas, lo cual repercute en un aumento de costes. Se amplía también el abanico de especialidades que ofrece el sistema cooperativo y son cada vez más las cooperativas de enseñanza que ofrecen el Bachillerato y la Formación profesional. (Alfonso Sánchez, 2002)

No sólo esto sino que también se introducen en la enseñanza infantil que si bien no es obligatoria en el sistema educativo es una necesidad social que lleva a las Autonomías a 
fomentar la creación de cooperativas especializadas, o llegar a acuerdos con las existentes para que amplíen sus objetivos. En este caso, para las cooperativas significa una fuente de ingresos que les permite mantenerse o ampliar el espectro de sus ofertas.

Por otra parte se consolidan los aspectos legales de las cooperativas que reciben las calificaciones definitivas, lo que estabiliza su situación legal y social. También en esta etapa se produce un incremento de los cooperativistas con titulaciones superiores, lo que les permite ampliar el marco de las enseñanzas y entrar a formar parte de las cooperativas a profesionales jóvenes, que sustituyen a los jubilados que habían sido los fundadores de muchas de las cooperativas, con lo que se rejuvenece el cuadro profesional.

También es cierto, por último, que la situación financiera de las cooperativas especialmente en los primeros años del siglo era buena. Las inversiones fundacionales y la capitalización que por precaución adoptaron muchas de ellas en vez de repartir los beneficios entre los cooperativistas, implicó que la economía de la mayoría de las cooperativas asentadas fuera entre buena y muy buena.

Hoy día, si bien la situación económica no es tan buena, el cooperativismo en la enseñanza, es una realidad que, en España, puede evidenciarse por las cifras que da la misma Unión Española de Cooperativas de Enseñanza (UECOE), cuyos propósitos son:

“.- Crear empleo y mantener el puesto de trabajo de los socios y trabajadores, en primera instancia y la dignificación del mismo, a largo plazo.

-- Entender la gestión empresarial como indispensable, procurando una profesionalización de los equipos directivos.

-- Promover y fomentar la cultura emprendedora desde y en el aula de los centros asociados.

-- Aportar soluciones a problemas concretos de la educación.

-- Construir el proyecto pedagógico junto al proyecto empresarial y éstas actualmente se encuentran en una situación óptima para brindar alternativas válida al mundo educativo." (UECOE).

Los cuales son, en general, principios fundamentales del cooperativismo. El éxito de los mismos en España, se manifiestan por los números que da la misma institución respecto a 2012: 
.- Cooperativas de enseñanza y centros educativos de economía social: 606.

.- Trabajadores (socios y asalariados): 26.751.

.- Alumnos escolarizados: 274.769.

.- Millones de euros de facturación: $748 .^{7}$

El cooperativismo en la enseñanza según estas cifras, presentan un buen posicionamiento en el sector en un momento en que el mismo está atravesando una crisis que más que profesional ya es social, probablemente también estructural y, desde luego, económica. Esta crisis afecta a la enseñanza en conjunto, es cierto, pero de manera más incisiva a las expectativas profesionales de los enseñantes que ven mermadas, cuando no anuladas, sus expectativas futuras y su calidad de vida profesional. Con este panorama, las cooperativas de enseñanza se plantean como una solución por lo menos a los puestos de trabajo. Un ejemplo de esta tendencia está en algunas especialidades de la enseñanza que ante los problemas planteados se inclinan por salvar puestos de trabajo por medio de la creación de cooperativas de profesores, como es el caso de la enseñanza de ELE.

\section{ORGANIZACIÓN Y ESTRUCTURA DE LAS COOPERATIVAS EN LA ENSEÑANZA}

Dentro de las sociedades cooperativas de la enseñanza aparecen dos tipos de estructuras: una estructura orgánica y una estructura administrativa. La primera la conforman los elementos que usan los socios para gestionar la empresa. Los cargos directivos en este tipo de empresas suelen estar controlados por los socios a través de elecciones entre ellos para cubrirlos.

En segundo lugar la estructura administrativa que depende de la primera, son los elementos de la organización que desarrollan las instrucciones de la dirección y pueden ser o no socios.

\subsection{La estructura orgánica}

La ley de 27/1999 de 16 de julio de Cooperativas, cuya vigencia termina el 23 de Agosto de 2013 según el encabezado de dicha Ley, dice en su Capítulo IV, de los órganos

\footnotetext{
${ }^{7}$ Sólo a efectos de comparar las magnitudes según la Confederación empresarial Española de la Economía Social (CEPES), en el primer trimestre de 2012, que son los datos recogidos más recientes, en España existían 22.022 cooperativas con un total de 298.298 empleos. Datos facilitados por la propia CEPES. http://www.cepes.es/pagina_cepes $=10$
} 
sociales, que estos son: La Asamblea General, El Consejo Rector y la Intervención, aun cuando la sociedad cooperativa puede libremente ampliarlos con un Comité de Recursos y otros órganos que consideren necesario de carácter consultivo o asesor ${ }^{8}$.

Pero la situación no es tan sencilla como parece, dado que las cooperativas están sujetas también a la legislación autonómica que establece normativas y órganos sociales diferentes, aun cuando algunos de ellos sólo difieren en el nombre, ya que se trata de los mismos con otra denominación. Aun cuando también hay algunos que son nuevos. A esta problemática se suman los órganos obligados por la Ley Orgánica reguladora del Derecho a la Educación y la Ley Orgánica de la Participación, la evaluación y el Gobierno de los Centros Docentes, por cuanto las cooperativas están sujetas a las dos normativas. Estas últimas exigen, en el caso que la cooperativa esté concertada con las administraciones públicas, un Consejo Escolar, un Claustro de profesores y la figura de un Director.

No hay que obviar que la Ley de referencia establece en su art. 78 el número de socios en un mínimo de 3, lo cual nos parece un número muy reducido para una cooperativa. Otro artículo que induce a reflexión es el 12 que establece el tipo de socios que puedan participar en una cooperativa y son tanto las personas físicas como las jurídicas, públicas y privadas e, incluso, las comunidades de bienes. En cualquier caso remite a los estatutos en los que se establecerán los requisitos necesarios para participar como socio en una cooperativa en general. $^{9}$

También es de aplicación, por lo menos en parte, la Ley de 5/2011, de 29 de marzo de Economía Social, cuyo objetivo primero es establecer "un marco jurídico común para el conjunto de entidades que integran la economía social" y en el capítulo $4^{\circ}$ define las entidades de economía social como las que defiende y practican la "primacía de las personas y del fin social sobre el capital”. (Paniagua Zurera, 2011)

\footnotetext{
${ }^{8}$ Las cooperativas aparecen en la Constitución Española, artículo 129.2 "Los poderes públicos promoverán eficazmente las diversas formas de participación en la empresa y fomentarán, mediante una legislación adecuada, las sociedades cooperativas. También establecerán los medios que faciliten el acceso de los trabajadores a la propiedad de los medios de producción"

${ }^{9}$ 1. En las cooperativas pueden ser socios, en función de la actividad cooperativizada, tanto las personas físicas como jurídicas, públicas o privadas y las comunidades de bienes.

2. Los Estatutos establecerán los requisitos necesarios para la adquisición de la condición de socio, de acuerdo con lo establecido en la presente Ley.
} 


\subsection{Estructura administrativa}

Sin embargo, la estructura administrativa de una empresa cooperativa de enseñanza es más o menos igual que cualquier otra empresa, que tiene por objeto la organización de la gestión y las relaciones formales que mantiene el entramado empresarial. Cierto que uno de los factores fundamentales de toda organización administrativa es una jerarquía que garantice el control de los trabajadores y las funciones de cada uno, del trabajo y sus resultados y de los flujos de caja de la organización por los directivos de la misma. Lo cual en una sociedad cooperativa en que la mayor parte de los productores son a la vez socio, crea algunos problemas de relación, psicológicos y de jerarquía.

El Consejo Rector de la cooperativa tiene entre sus atribuciones nombrar al Director. A partir de este mando administrativo se organiza toda la estructura. Es quien está encargado de garantizar el logro de los objetivos fijados por los socios de la cooperativa a través de sus órganos, especialmente de la Asamblea General. Estos órganos administrativos suelen ser cubiertos por profesionales, lo cual no implica que en determinados casos cubran los puestos los propios socios de la cooperativa.

Si bien, como hemos dicho, la organización administrativa es pareja a cualquier empresa, no hay que dejar de lado que se trata de una cooperativa de enseñanza con particularidades propias, tanto desde la perspectiva económica como de la jurídica.

Respecto a la primera, en el aspecto administrativo, las cooperativas no tienen gerentes en el sentido estricto sino directivos subordinados a las decisiones de los órganos de gobierno, que es lo mismo que decir que no disponen de autonomía para conducir la empresa libremente. Por otra parte los socios participan de las unidades administrativas y a la vez de los órganos sociales de control. A este respecto hay que destacar que una cooperativa de enseñanza se diferencia de los centros privados también de enseñanza en que la toma de decisiones es uno de los principios que proclama la Alianza Cooperativa Internacional: "Las cooperativas son organizaciones gestionadas democráticamente por los socios, los cuales participan activamente en la fijación de sus políticas y en la toma de decisiones".

En lo referente a la faceta educativa, hay que considerar la comunidad educativa: claustro de profesores, padres y alumnos como corresponsables en la consecución de los objetivos, los cuales también, de una forma u otra, participan en diversos grados y democráticamente en la toma de decisiones. 
Todo ello nos lleva a resaltar que implica un equilibrio de poderes entre diversos grupos que dificultan la gestión de los órganos de gobierno y también de la administración de las cooperativas de enseñanza. En cualquier caso no debe de olvidarse que las cooperativas de enseñanza participan, en primer lugar, de la ideología del cooperativismo y, por lo tanto, de los principios que lo conforman, entre ellos el quinto que dice: "Las cooperativas proporcionan educación y formación a los socios, a los representantes elegidos, a los directivos y a los empleados para que puedan contribuir de forma eficaz al desarrollo de sus cooperativas.” Lo cual implica un objetivo ideológico además de una empresarial.

\subsection{Características}

Las cooperativas de enseñanza, como hemos dicho antes, presentan tipologías propias y diferenciadoras que tanto pueden ser de naturaleza jurídica, como organizativa o funcional, que derivan de las leyes que sobre cooperativas y sobre enseñanza les afectan directamente.

El cooperativismo en la enseñanza sigue formas jurídicas diferentes de los centros docentes públicos o privados que tienen sus propias normativas. Y son también diferentes a estos cetros en sus estructuras, por ejemplo presentan una burocratización inferior a los centros públicos, lo que supone una mayor agilidad en los procesos administrativos y menos costes. Respecto a los privados la mayor diferenciación reside en la dinámica de toma de decisiones, basándose el sistema cooperativo, como ya se ha dicho, en la conducción democrática de la empresa. Por otra parte los socios y los trabajadores en general de la cooperativa acceden a mayor información sobre las actividades de la empresa, su situación económica y los proyectos planteados que los trabajadores empleados de los centros privados.

La condición de socio y su capacidad de ejercer los derechos que confiere dicha posición, no está determinada por la aportación de capital sino por la actividad profesional que le compete en la cooperativa, en cambio, en un centro privado educativo, el derecho a voto de un socio depende de la cuantía de su aportación económica ${ }^{10}$. (Gadea, Sacristán y Vargas, 2009).

Por último los objetivos ideológicos de una cooperativa económica, aun cuando en el siglo XXI no son tan altruistas como en tiempos de los socialistas utópicos, se orientan a ofrecer un mejor servicio que se traduce en una mejor educación, una mayor integración de

\footnotetext{
10 Las cooperativas de enseñanza, aun cuando son centros privados, se consideran centros privados no convencionales por cuanto se diferencian de los centros privados convencionales.
} 
los padres y alumnos en un primer nivel, pero también del entorno social en que están ubicados. Su filosofía social es la de la ayuda mutua entre los socios que se implementa en ayudas económicas si son necesarias y personales cuando también lo son.

Cierto que hay varios tipos de cooperativas que cumplen objetivos diferentes aun cuando no divergentes. Por lo general se distinguen tres tipos referentes a la estructura: de trabajo asociado, en la que todos los socios son socios trabajadores; mixtas, en las que hay trabajadores socios y no socios y, por último, de consumidores y usuarios, que son uniones de socios de trabajadores y socios de consumo. En el caso de las cooperativas de enseñanza los padres de los alumnos.

Dada la especial sensibilidad que existe respecto a la educación en cualquier país, el nuestro no es una excepción, la labor de las cooperativas de la enseñanza se enmarca dentro de un sector en que es necesario compatibilizar las reglas de la empresa en busca de un beneficio y los valores sociales que obligan a un educador en particular y una empresa cuyo objetivo es la educación en general, a buscar un término medio entre el derecho de la empresa a unos beneficios y la obligación ética y jurídica de impartir una educación de calidad y los principios de la democracia que conforman el espíritu de la Constitución Española.

Las cooperativas de enseñanza están mejor dotadas de factores positivos para lograr este equilibrio. Por una parte, por lo general, no buscan tanto el beneficio como la seguridad de un puesto de trabajo en lo material, siendo frecuentes las reinversiones en materiales educativos y mejoras de las infraestructuras de buena parte de los beneficios.

Y, por otra, su ideología democrática y laica (sobre el $75 \%$ de los centros de enseñanza dependientes de cooperativas son laicos), así como los principios cooperativos, la hacen también muy adecuada como una alternativa civil y social proclive a introducir nuevos métodos de enseñanza en los que se aúnen todos los factores antes dichos, especialmente la calidad y la democracia como objetivos primeros de las sociedades cooperativas de enseñanza.

\section{TIPOS DE COOPERATIVAS DE ENSEÑANZA}

Si bien podemos tipificar las cooperativas de enseñanza no hay que olvidar, como hemos dicho antes, que cada una de ellas tiene sus peculiaridades que la particularizan. 
La Ley de Cooperativas recoge tres tipos: cooperativas de trabajo asociado, de consumo y de enseñanza. Aunque dada la definición que dicha ley proporciona, las cooperativas de enseñanza tienen necesariamente que ser también de trabajo asociado o de consumo, si los socios son docentes en el primer caso o padres o alumnos en el segundo. El problema se plantea por cuanto la misma ley establece normativas diferentes a aplicar si la sociedad de enseñanza es de uno u otro tipo. En definitiva la tipología que la ley citada reiteradamente propone es:

.- Cooperativas de proveedores de educación.

.-Cooperativas de trabajo asociado en la educación.

.- Cooperativas de consumidores de educación.

-- Cooperativas de padres de alumnos

-- Cooperativas de alumnos mayores de edad

.- Cooperativas de alumnos menores de edad

.- Cooperativas de servicios

.- Cooperativas integrales de educación.

\section{PROBLEMAS CONCRETOS DE LAS COOPERATIVAS DE ENSEÑANZA}

Como hemos dicho recientemente, las cooperativas de enseñanza ofertan una diferencia frente a las instituciones de enseñanza clásicas en nuestro sistema educativo, las escuelas públicas y las privadas. Una diferenciación que plantea un enfrentamiento directo, en lo ideológico, con el modelo privado por cuanto hasta hace muy poco tiempo estaba controlado por colegios de tipo religioso. Aunque en los últimos años del siglo XX y primeros del actual, el panorama de los centros de enseñanza españoles ha variado mucho en lo referente a la ideología de los centros privados.

Las cooperativas de la enseñanza ofrecen, desde su aparición, una nueva oferta a través de un nuevo modelo empresarial, funcional y educativo, por lo general dentro de la posición aconfesional y los principios democráticos. La doble condición de los cooperativistas como docentes y socios implica una forma de entender la educación y, a la vez, una forma de trabajar diferentes que, en puridad, está más cerca de la visión pública que de la privada en el momento actual.

Esto no quiere decir que todas las cooperativas de la enseñanza son igual ni desde la perspectiva estructural ni desde la humana, ya que tanto en sus relaciones exteriores como en 
las interiores cada una de ellas tiene su personalidad particular, que depende de las personas que la integran pero también del modelo o tipo de cooperativa que han querido implementar.

En las cooperativas conformadas por socios docentes, por lo general la docencia se estructura como el objetivo final pero también como el factor que informa la organización de toda la empresa. La problemática que plantean suele ser una excesiva orientación hacia la pedagogía y aunque parezca lo más razonable, no pueden olvidarse los aspectos sociales de la cooperativa y los fines ideológicos que la sustentan. Las cooperativas asumen la educación por valores que pretenden preparar al alumno no sólo para superar unas pruebas selectivas en mayor o menor medida, sino también y preferentemente a vivir, convivir y trabajar en equipo. Lo cual implica enseñar al alumno habilidades de relación, comunicación, tratamiento de la información, esfuerzo y empatía (Fernández, 2000).

En las cooperativas de tipo mixto, intervienen tres grupos claramente diferenciados: los profesionales, entre los que podemos distinguir dos sub-grupos, los docentes y los no docentes; los alumnos, que se confunden con sus padres o tutores legales, que en definitiva son los que toman las decisiones y aquellos que sin ser docentes, no docentes o alumnos, participan, tanto como personas físicas como jurídicas, de forma explícita en el proyecto y que pueden ser entidades públicas, patronados, antiguos alumnos y cualquier otra institución que de alguna manera esté involucrada en la cooperativa. En este caso, la problemática se circunscribe a lograr que estos tres grupos se pongan de acuerdo en un proyecto común, lo cual no es fácil, dado que cada uno de ellos, además de sus tendencias cooperativistas, defiende puntos de vista particulares (Fernández, 2000).

Fernández Guadaño (1999 y 2000) se inclina por los conceptos de sociedades cooperativas de proveedores de educación o sociedades cooperativas de consumidores de educación, y no deja de tener razón a nuestro juicio, por cuanto en la estructura de la sociedad en general unos actúan como proveedores, profesores y personal administrativo, y otros como consumidores, padres y tutores. Dentro de esta línea también se encuentra Paz Canalejo (1878), que realiza un amplio análisis de los tipos de cooperativas de enseñanza.

Podemos decir que los problemas planteados hasta aquí son internos, pero también se plantean problemas externos. Las cooperativas de enseñanza se enfrentan en el siglo XXI con nuevos planteamientos globales que afectan a la empresa y a la educación en general que cuanto menos pueden calificarse de novedosos. Las nuevas tecnologías han potenciado 
aspectos de la enseñanza y, también, la competitividad dentro del sector. Nuevos modelos de enseñanza, directos o virtuales, presenciales o no, se ofrecen en un mercado saturado que obliga a encontrar nuevos planteamientos y nuevos esquemas que permitan posicionarse a las empresas en general y las cooperativas en particular. (Fuente Viñas, 2002).

Todo ello, competencia, nuevas tecnologías y nuevos planteamientos, implica una formación continua de los profesionales del sector y más en una empresa cuyos objetivos y planteamientos ideológicos se dirigen a la mayor calidad y eficacia en la enseñanza. Las cooperativas están conformadas por socios con los mismos derechos y deberes, pero esto no significa mucho si la oferta es calidad; lo que necesitan las cooperativas, como cualquier otro centro de enseñanza que quiera competir con ventajas en el mundo actual, es contar con profesionales cualificados, sean socios o no. Pero si éstas están formadas por socios, es evidente que son ellos los que tienen que ofrecer la calidad y eficacia, por lo cual deben seguir un reciclaje constante.

Cierto es que el cooperativista se enfrenta a dos funciones: la de socio de la cooperativa y la de docente, o aquella que tenga asignada, una de ellas debe realizarla cuando termina con la otra, lo que le obliga a una mayor dedicación por una parte y a adquirir conocimientos de gestión y dirección de empresa por otra, para cumplir sus responsabilidades como socio cooperativista. Y, desde luego, debe seguir formación continua en los dos aspectos de sus funciones de manera que no se resienta su labor en cualquier de ellas.

Uno de los problemas que se plantearon a finales de la década de los noventa del siglo anterior y parte de la primera del siglo XXI fue la desaceleración demográfica que implicaba menos alumnos y problemas económicos para la cooperativas más que para los centros privados, dado que los socios de aquellas eran a la vez profesionales, lo cual significa que sea muy difícil recurrir a las soluciones que tienen las empresas privadas no cooperativas para reducir el personal. Sin embargo este problema en la actualidad se ha estabilizado; sea por causa de la inmigración o por otra lo cierto es que en el período 2011-2012 y en el 2012-2013 los alumnos matriculados en enseñanza no universitaria fueron:

$\begin{array}{lll}\text { Año } & \text { Centros Públicos } & \text { Centros Privados. } \\ 2011-2012 & 5.394 .203 & 2.529 .090 \\ 2011-2013 & 5.470 .312 & 2.536 .064^{11}\end{array}$

${ }^{11}$ Sólo a efectos comparativos ofrecemos los siguientes datos provenientes de la misma fuente: 
Porcentualmente el aumento es del $1 \%$ en la enseñanza pública y el $0,3 \%$ en la privada, por lo que puede hablarse de un estancamiento demográfico. (Datos del Ministerio de Educación, Cultura y Deporte) ${ }^{12}$

Por último, resumimos lo que hemos dicho respecto a la problemática del sector en dos problemas que nos parecen los más importantes a los que se enfrentan las cooperativas de enseñanza. En primer lugar en las sociedades cooperativas integrales, como ya hemos dicho, presentan intereses contrarios entre el grupo profesional y los padres de los alumnos. En este caso, la cuestión es evitar que uno de los dos grupos pueda imponerse al otro. Y es más probable que sea el segundo, el de los padres, porque son muchos más. Esta posibilidad ya se prevé en la Ley española que establece la posibilidad de porcentajes de votos ${ }^{13}$.

En segundo lugar, en las cooperativas de trabajo asociado en la educación se dan dos socios proveedores: los docentes y el personal administrativo o de servicios, los cuales pueden y suelen ser asalariados pero también pueden ser socios. Esto suele ocasionar problemas porque las visiones de la empresa son distintas. La solución viable es que el reglamente interno especifique muy bien las funciones, derechos y deberes de cada uno de ellos.

No son los únicos problemas que se plantean en una cooperativa de la enseñanza, pero sí los más conflictivos. En cualquier caso, las cooperativas tienen la ventaja que sus problemas se pueden discutir entre socios y solucionarlos entre iguales, lo que no ocurre en los centros privados convencionales, ni siquiera en los públicos.

\section{EL CASO DE GREDOS SAN DIEGO COOPERATIVA: UN EJEMPLO DE COOPERATIVA DE ENSEÑANZA}

Un ejemplo de buen funcionamiento de una cooperativa de enseñanza es Gredos San Diego Cooperativa de Enseñanza, que fue fundada en abril de 1985 por 18 trabajadores, cuando el propietario del colegio se jubiló, con el objetivo de impedir el cierre y conservar sus puestos de trabajo. Por una serie de causas que se entrelazaron, en aquellos años cerraron muchos colegios en nuestro país. Por un lado fueron causas

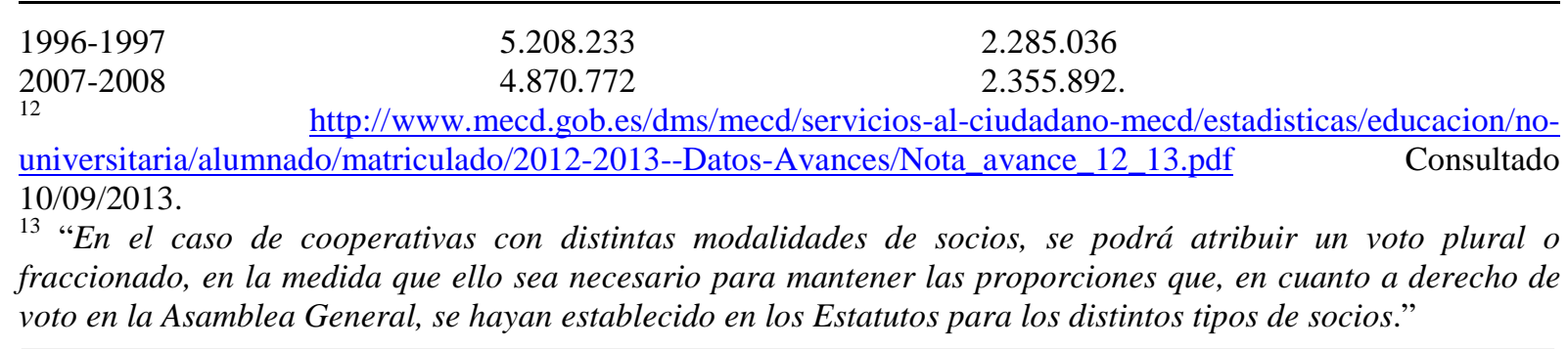


extrínsecas a la educación, como el descenso de la natalidad que incidía, años después, en la demanda de plazas y, por otro, la nueva Ley Orgánica del Derecho a la Educación que exigía unos mínimos en cada una de las instituciones, que muchas de ellas no podían cubrir.

No era un futuro muy prometedor al que se enfrentaron los 18 socios que se constituyeron en una empresa de enseñanza de trabajo asociado, grupo que incluía docentes y no docentes unidos no sólo por una necesidad de conservar sus puestos de trabajo sino también por una vocación docente y social. El primer centro cultural estuvo situado en la Avenida de San Diego, en el Puente de Vallecas de Madrid, en el límite en que confluyen tres barrios con solera y personalidad: San Diego de Puente de Vallecas, Palomeras Baja y Barrio de Picazo, los tres de tradición obrera y luchadora.

La situación geográfica marca buena parte de la ideología y características del centro en los primeros años y de los primeros alumnos, especialmente de los obreros de los alrededores que se matricularon en el bachillerato nocturno, con el objeto de promocionarse a través de los estudios. Mientras que el bachillerato diurno se nutría de hijos de obreros de la construcción, fábricas de la barriada o trabajadores de servicios municipales, además de una buena proporción de alumnos procedentes de la etnia gitana.

El currículo, la ideología, el cuadro docente y, en general, los socios de la cooperativa impusieron un carácter social a su trabajo sobre la base del modelo de Economía Social que propugnaban. En 1994 se inauguró el Colegio Gredos San Diego Vallecas, que cumplía ya con todas las condiciones reclamadas por la LOGSE. En 2000 abrió sus puertas Gredos San Diego Moratalaz y en 2005 Gredos San Diego El Escorial. La expansión de la Cooperativa continuó en los años posteriores con la creación del Grupo GSD cuyos objetivos han estado, desde el primer momento, ligados a la educación, las acciones sociales y culturales, y la propagación de los valores cooperativos especialmente dentro del marco de la enseñanza.

En la actualidad el grupo cuenta con 1.400 trabajadores de los cuales 922 son socios, entre docentes y no docentes y la incidencia del Grupo en la Enseñanza no universitaria de la Comunidad Autónoma de Madrid es del 5\% del total. Cuenta con otros colegios; a los mencionados se le sumaron posteriormente Colegio Gredos San 
Diego Las Suertes, en 2007, Colegio Gredos San Diego Las Rozas en 2008 y el Colegio Gredos San Diego Alcalá, fundado en 2007 y Colegio Gredos San Diego Guadarrama. En 2012 los alumnos del Grupo fueron más de 13.000 y sirve 10.000 comidas todos los días. Uno de los aspectos que destacan y se insertan también en la economía social es que entre sus trabajadores y socios hay representadas 22 nacionalidades y un 71,54\% de ellos son mujeres que ocupan el 52,72\% de los puestos directivos.

\subsection{La educación}

Los niveles educativos que se implementan en la Cooperativa son:

.- Escuela Infantil.

.- Primaria y secundaria obligatoria.

-- Bachillerato.

.- Formación profesional.

Gráfico 2. Situación cuantitativa de la Cooperativa Gredos San Diego.

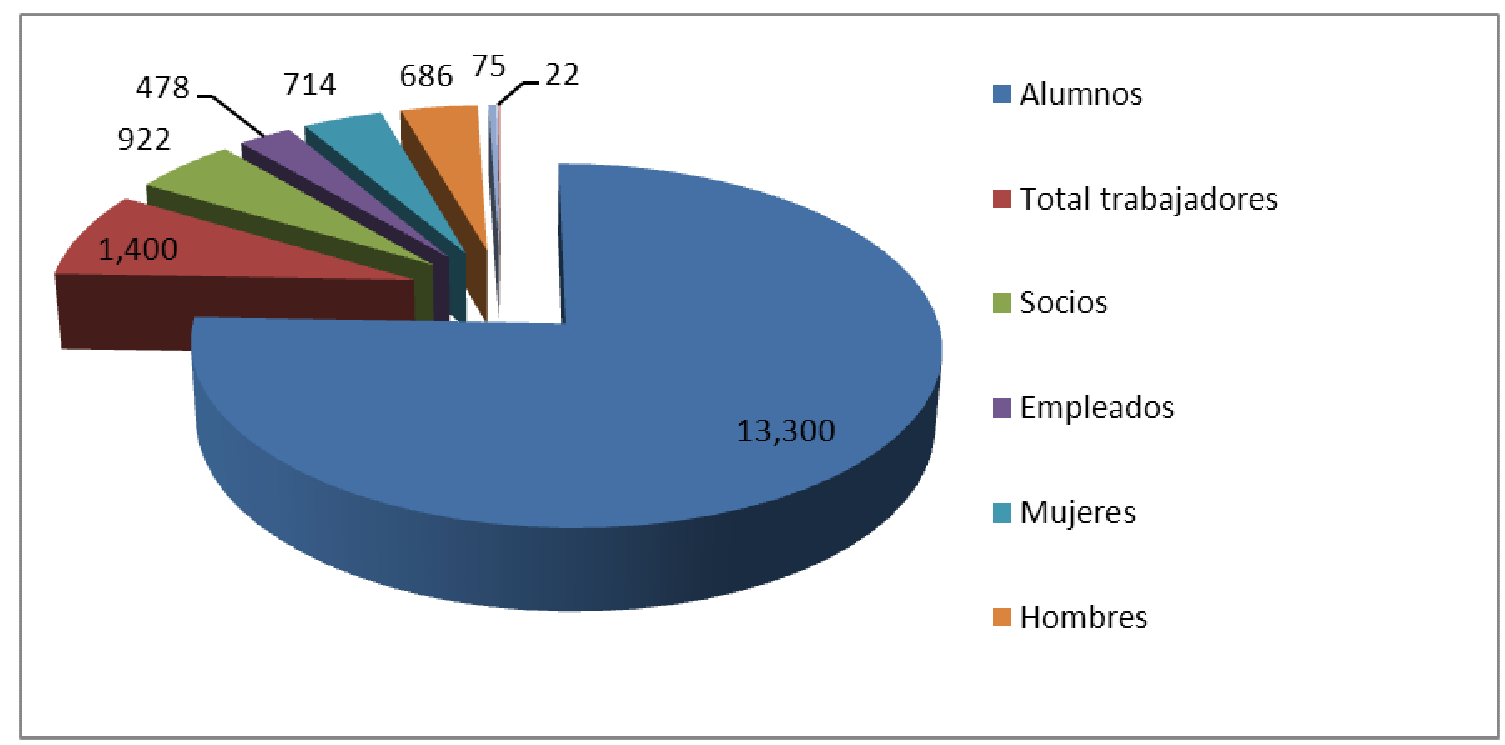

Fuente: Elaboración propia

En resumen se imparten todos los niveles educativos desde la primaria hasta los 18 años. La educación sigue el sistema concertado desde el $2^{\circ}$ ciclo de Educación Infantil hasta los 16 años y la privada el $1^{\circ}$ Ciclo de Educación Infantil y el Bachillerato. Las Formación profesional se imparte en el Grado Medio y el Grado Superior en el modelo concertado. Además proporciona otros ciclos formativos que van desde Escuela de Música hasta gabinete psicológico, médico, servicios de comedor y deportivos. 


\subsection{Responsabilidad social corporativa}

La actuación social ha sido, desde el primer momento, una constante en el pensamiento pedagógico de la cooperativa. Los principios cooperativos reflejados en la honestidad, solidaridad, tolerancia y democracia han significado unos claros objetivos en la actuación de todos los participantes en el proyecto. Y lo han sido en las dos vertientes básicas de la cooperativa: por una parte respecto al personal al que se le han inculcado los valores esenciales que implican un cometido social en sus actividades profesionales $\mathrm{y}$, por otra, en el contenido educativo de los programas que se implementan.

No obstante es cierto que en este momento las líneas de actuación social son varias; han sido de especial relevancia los aspectos de la educación ambiental con la promoción de actividades de sensibilización y programas como las Ecoescuelas que se iniciaron en 2004.

Más moderno es el Plan Integra que promueve la integración de las personas con discapacidad intelectual en los Centros GSD. El objetivo de la iniciativa es mejorar el nivel de vida de estas personas y potenciar sus capacidades. Otras actividades son la prevención de desigualdades en las escuelas o las Aulas de Enlace para la mejor adaptación de los alumnos extranjeros a los Colegios del grupo, de manera que puedan incorporarse sin traumas al sistema educativo español o las acciones para la integración de alumnos con discapacidades a través de las aulas TGD, etc.

Todo ello comporta un compromiso claro con los principios del cooperativismo, entre ellos la reinversión de los beneficios en nuevas actividades que promocionen la relación del grupo con la sociedad en la que se ubica y la especial sensibilidad con el entorno.

Respecto a la estructura organizativa del grupo destaca el poco interés que se ha puesto en la tendencia, casi general de las cooperativas, por el asamblearismo que, en el mejor de los casos, es poco operativo en una cooperativa como el Grupo Gredos.

La Asamblea General es el máximo órgano decisorio, aun cuando delega muchos de sus poderes en el Consejo rector, lógicamente más reducido el cual define las líneas maestras de las actuaciones que serán ejecutadas por un Presidente Ejecutivo 
o un Gerente, que no pertenecen al Consejo Rector. La integración de todos los trabajadores, sean docentes o no, en el proceso de dirección y organización de la cooperativa ha dinamizado la gestión de la misma obteniendo brillantes resultados.

Gráfico 3. Situación de la Cooperativa Gredos San Diego.

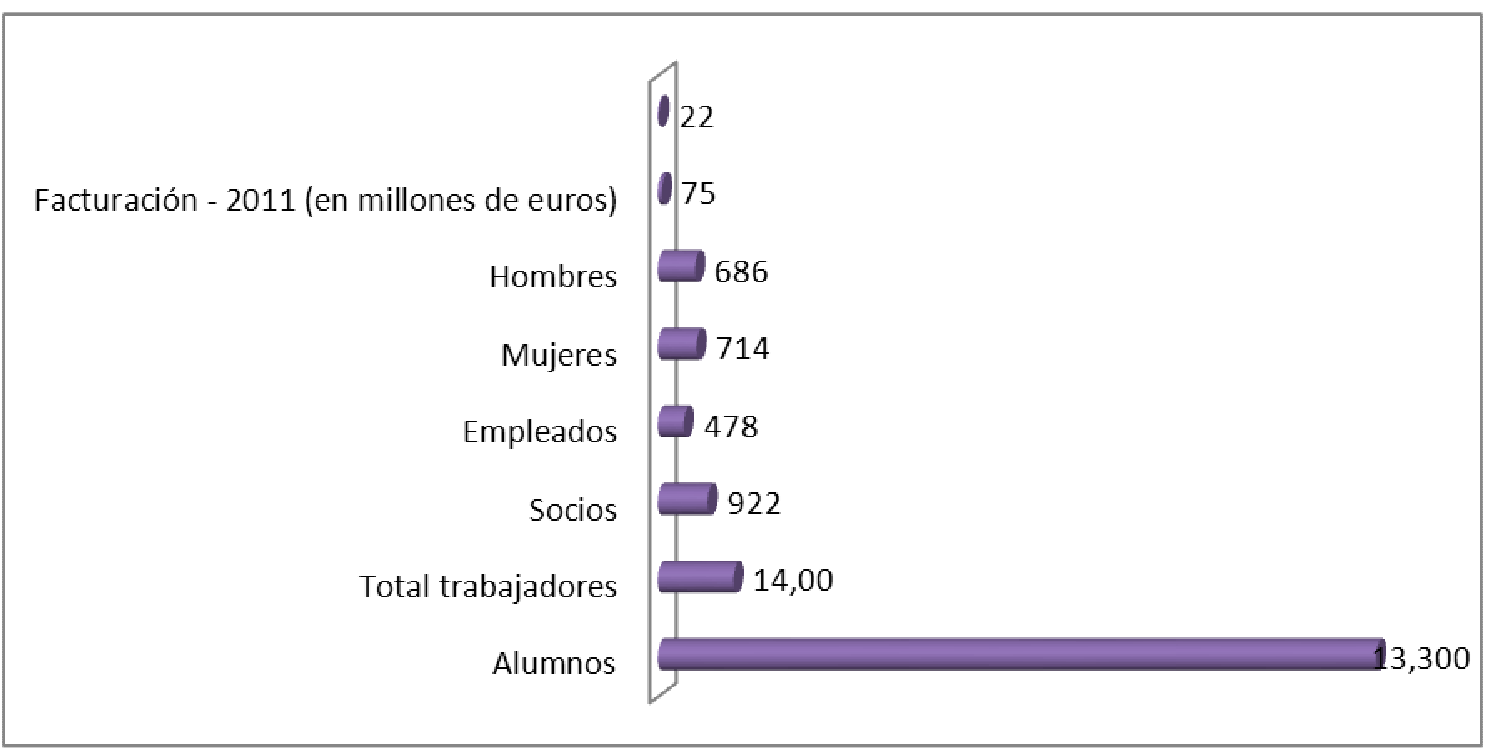

Fuente: Elaboración propia

A la vista de este caso podemos concluir afirmando que la principal característica del Grupo Gredos San Diego Cooperativa es su ideología progresista, que proviene de los primeros años de su desarrollo a partir tanto del hecho fundacional, la compra por 18 trabajadores de la enseñanza constituidos en cooperativa, como la ubicación de la escuela en un entorno obrero por excelencia. Los dos factores determinaron su historia como centro de estudios que cubría las necesidades formativas de los obreros que querían progresar a través de los estudios y de sus hijos.

Estos inicios y los valores que asumieron, respeto a la dignidad humana y fomentar los valores científicos y humanistas, se han mantenido y potenciado a lo largo de estos años hasta el presente. Durante todo el periodo ha mantenido su vocación pedagógica y su expansión ha estado asociada a las necesidades sociales, deportivas y culturales de los sectores obreros y de clase media.

\section{CONCLUSIONES}

Las cooperativas de educación son, a nuestro juicio, uno de los más claros modelos futuros de empresas educativas, tanto por sus ideales como por su adscripción a la moderna 
mentalidad sobre la enseñanza en el sector privado. Es muy posible que en un futuro no muy lejano la educación privada se enfrente con problemas docentes pero los especialmente graves serán económicos. Posiblemente también a la corta afecten menos a los centros religiosos que a los no religiosos, aun cuando a la larga también los religiosos tendrán que reestructurar sus metodologías y objetivos. Realmente ya lo están haciendo en este momento.

Los problemas con que se enfrentan las sociedades cooperativas de la enseñanza, a nivel interno, son de fácil solución en tanto y cuanto no parecen tan importantes como para afectar esencialmente a un proyecto. Entre ellos uno que siempre ha sensibilizado a los cooperativistas de la enseñanza, la reputación de los centros y del profesorado. A este respecto destacan los estudios de Martínez León, Olmedo Cifuentes y Zapata Conesa (2013)

Por otra parte, los problemas externos son a los que se enfrentan todas aquellas empresas que se dedican a la enseñanza fuera de su estructura; los centros docentes privados convencionales son empresas cuyo objetivo primero es obtener beneficios, no es el caso de las cooperativas, aun cuando sea uno de los objetivos, pero no el primero.

En este contexto, independientemente de la evolución de la educación, las nuevas tecnologías o la sociedad de la información y del conocimiento, las sociedades cooperativas cuyos principios se centran en ofrecer el más eficiente servicio y la mejor calidad, o sea buscar la excelencia, tienen una ventaja competitiva en el sector. Por una parte su oferta será más interesante en el mercado futuro y, por otra, como ya hemos indicado, al no tener socios capitalistas que reclamen beneficios, puede destinar más capital a las inversiones.

En el caso concreto de Gredos San Diego Cooperativa hemos comprobado que su éxito docente, social y económico, atestigua que un proyecto bien estructurado y con unos objetivos claros, permite mantener y expandir una sociedad cooperativa de la educación siempre que se mantengan los principios cooperativistas.

Esta mayor competitividad en el sector es justamente lo que hemos planteado como hipótesis: el futuro de la enseñanza privada pasará a depender de las cooperativas de enseñanza en su mayor parte, por lo que este tipo de centros deberá ser promovido por el Estado como gestor de la enseñanza nacional y la propia sociedad como usuaria de los servicios de las cooperativas de enseñanza. 


\section{BIBLIOGRAFÍA}

ACI (1996) Declaración de Identidad de la Alianza Cooperativa Internacional. Journal International Association of Cooperative Law, $\mathrm{n}^{\circ} .25$, pp. 21-26.

ALFONSO SÁNCHEZ, R. (2002) La transformación de la sociedad cooperativa. Murcia: Universidad de Murcia.

ARANGO JARAMILLO, M. (2005) Manual de cooperativismo y economía solidaria. Bogotá: Unión Cooperativa.

FERNÁNDEZ GUADAÑO, J. (1999) Las sociedades cooperativas en la educación ante la nueva concepción del Estado en la provisión de servicios educativos. REVESCO. Revista de Estudios Cooperativos n ${ }^{\circ} .67$, pp. 71-88.

FERNÁNDEZ GUADAÑO, J. (2000) La prestación del servicio educativo por las sociedades cooperativas frente a los centros públicos y privados convencionales. Revista de Estudios Empresariales. núm. 20, pp. 383-402.

FERNÁNDEZ GUADAÑO, J. (2000) La realidad actual de las Sociedades Cooperativas en la Educación. REVESCO. Revista de Estudios Cooperativos nº. 71, pp. 55-76.

FERNÁNDEZ GUADAÑO, J. (2003) La participación, factor determinante de la calidad educativa de los centros docentes educativos. REVESCO. Revista de Estudios Cooperativos. $\mathrm{n}^{\mathrm{o}}$. 70, pp. 35-57.

FUENTE VIÑAS, A. M. et al. (2002) Las cooperativas de enseñanza como tercera vía dentro de nuestro sistema educativo: las cooperativas de trabajo asociado. Percepciones de sus directivos. Salamanca: Ediciones Universidad de Salamanca.

GADEA, E.; SACRISTÁN, F. y VARGAS VASSEROT, C. (2009) Régimen jurídico de la Sociedad Cooperativa del Siglo XXI. Realidad actual y propuestas de reforma. Madrid: Dikinson.

MARTÍNEZ LEÓN, I.; OLMEDO CIFUENTES, I. y ZAPATA CIFUENTES, J. (2013) Reputación percibida por el profesorado de las cooperativas de educación: mediación e influencia. Ciriec-España Revista de Economía Pública, Social y Cooperativa, núm. 77, pp. 237-260.

MARX, C. (1973) El Capital Tomo I. La Habana: Editorial de Ciencias Sociales.

MIJAILOV, M. (1984) La Revolución Industrial. Buenos Aires: Guayas.

MORENO HERNÁNDEZ, A. (1992) Situación y perspectivas de la Cooperativas de Enseñanza. En V Congreso estatal de las Cooperativas de enseñanza. Sevilla: UECO. 
ORMAETXEA, J.M. (2003) Didáctica de una experiencia empresarial. El cooperativismo de Mondragón. Mondragón: Saiolan.

PANIAGUA ZURERA, M. (2011) Las empresas de la economía social. Más allá del comentario a la Ley 5/2011, de economía social. Madrid: Marcial Pons.

PAZ CANALEJO, N. (1978) Consideraciones sobre las llamadas "Cooperativas de Enseñanza”. Revesco, núm 44, pp. 3-32.

RODRÍGUEZ GONZÁLEZ, A. y ORTEGA ÁLVAREZ, A. (2009) Empresas de economía social y fomento del empleo. Una perspectiva jurídico-económica. Rev. Servicios Sociales y Política Social. ${ }^{\circ} 77$, pp. 65-82.

UNESCO (1950): Las cooperativas y la educación fundamental París.: Unesco. 